DIW BERLIN

Discussion Papers

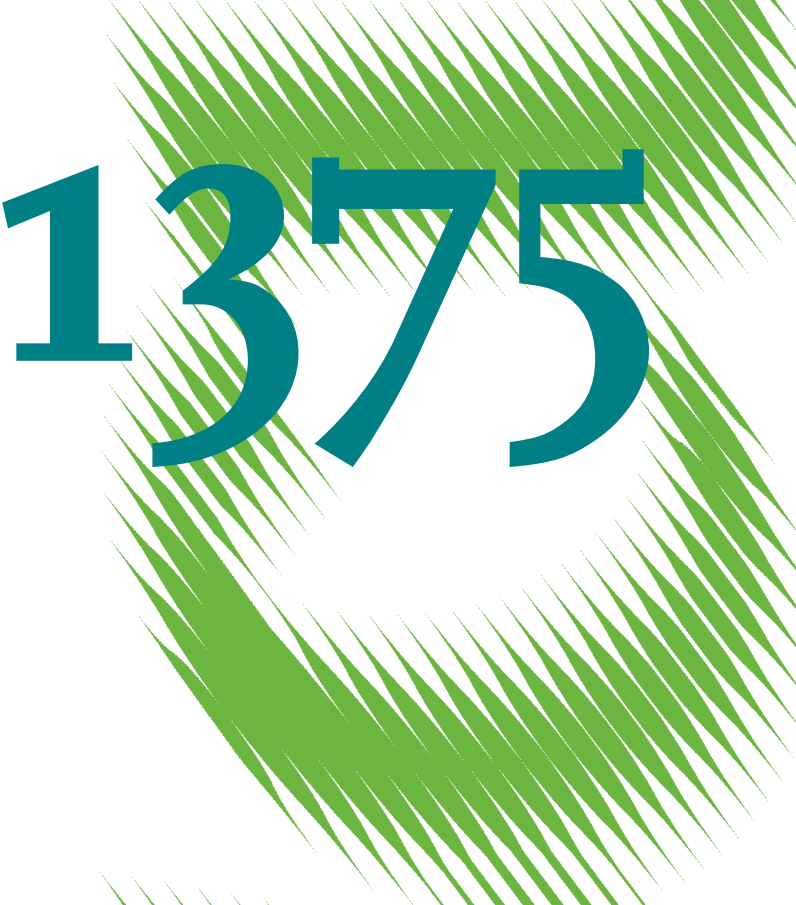

Are There Bubbles in Stock Prices? Testing for Fundamental Shocks 
Opinions expressed in this paper are those of the author(s) and do not necessarily reflect views of the institute.

IMPRESSUM

(C) DIW Berlin, 2014

DIW Berlin

German Institute for Economic Research

Mohrenstr. 58

10117 Berlin

Tel. $+49(30) 89789-0$

Fax +49 (30) $89789-200$

http://www.diw.de

ISSN print edition $1433-0210$

ISSN electronic edition 1619-4535

Papers can be downloaded free of charge from the DIW Berlin website:

http://www.diw.de/discussionpapers

Discussion Papers of DIW Berlin are indexed in RePEc and SSRN:

http://ideas.repec.org/s/diw/diwwpp.html

http://www.ssrn.com/link/DIW-Berlin-German-Inst-Econ-Res.html 


\title{
Are there Bubbles in Stock Prices? Testing for Fundamental Shocks
}

April 2014

\author{
Anton Velinov \\ DIW Berlin and European University Institute, Florence \\ Mohrenstr. 58, D-10117 Berlin, Germany \\ email: avelinov@diw.de \\ tel. +493089789460 \\ Wenjuan Chen \\ Free University Berlin \\ Boltzmannstr. 20, D-14195 Berlin, Germany \\ email: wenjuan.chen@fu-berlin.de \\ tel. +493083853240
}

\begin{abstract}
This paper investigates whether there are bubbles in stock prices. We do this using a previously studied structural vector autoregressive (SVAR) model claiming to distinguish fundamental and non-fundamental shocks to real stock prices. The SVAR model relies on an identification restriction in order to correctly label the shocks. We test this restriction by means of a Markov switching-SVAR (MS-SVAR) model in heteroskedasticity. Using data from France, Germany, Italy, Japan, the UK and the US we find that the restriction is rejected for Italy, supported at the $1 \%$ level for Japan and supported at least at the $5 \%$ level for the remaining countries. Several alternative specifications confirm the robustness of these findings. Using SVAR impulse responses and forecast error variance decompositions we further examine the structural shocks and confirm the shock labeling for Japan. Through historical decompositions we observe that stock prices tended to be undervalued throughout the 1970s and 1980s. This undervaluation corrects itself by the mid 1990s, after which stock prices tend to move in tandem with their fundamentals. We therefore find no evidence in favor of stock price bubbles in all the countries invested.
\end{abstract}

Key Words: Markov switching model, structural vector autoregression, heteroskedasticity, stock price fundamentals

JEL classification: C32 C34 E44 G12 


\section{Introduction}

There is a wide range of literature investigating stock prices and their relation to other macroeconomic variables. In particular, studies investigate whether there are bubbles in stock prices. We consider bubbles to be large positive deviations of stock prices from their fundamental values. For instance, observing the Dow Jones Industrial Average (DJIA) index in Panel (a) of Figure 1, we may come to the same conclusion as Binswanger (2004) that the rapid increase in US stock prices in the 1990s could have been due to a bubble component. This series is in absolute terms, which is the most common way it is depicted in the media and analyzed by analysts. However, observing the same series in real terms and in real log terms, (in Panels (b) and (c) respectively) we may be inclined to agree with Rapach (2001) that there is no stock price bubble present. In this paper we seek to answer the question of whether there are stock price bubbles by first testing whether we capture fundamental shocks to stock prices.

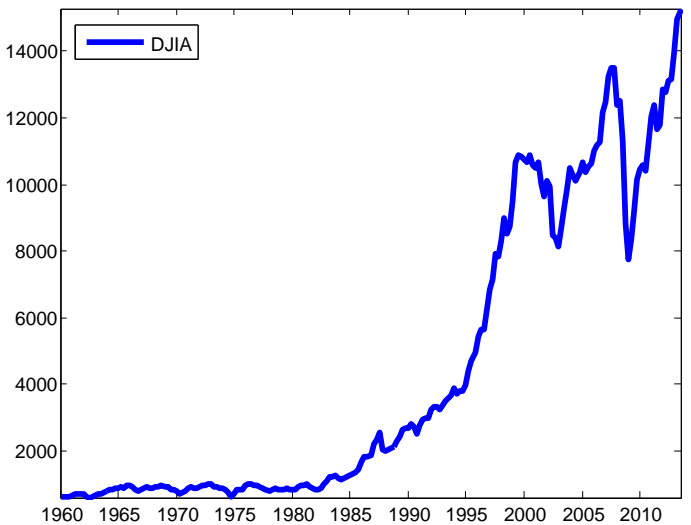

(a) Absolute series

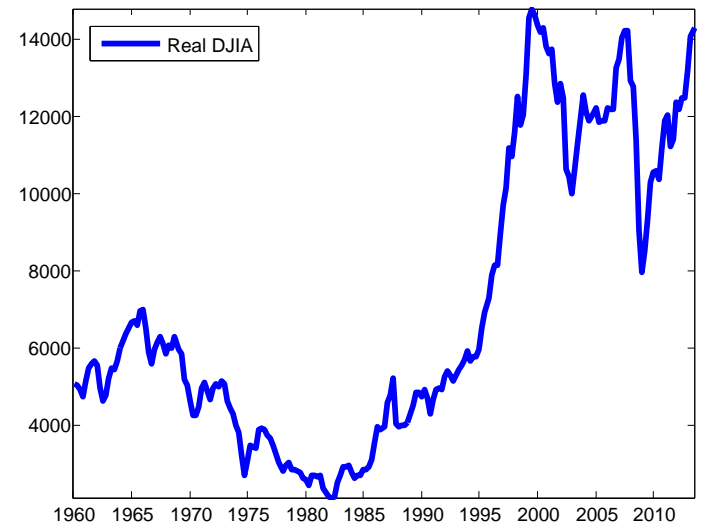

(b) Real series

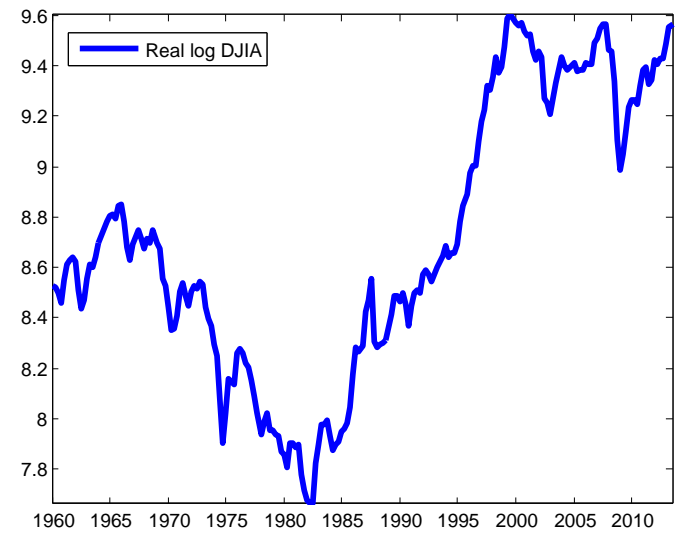

(c) Real log series

Figure 1: Dow Jones Industrial Average (DJIA) index. 
Vector autoregressive (VAR) or so-called reduced form models are commonly used in determining stock price fundamentals. Such models help draw inferences about economic relationships. However, due to their non-theoretical nature and correlated errors, their results could be subject to ambiguous interpretation. This is because there is no clear distinction of the shocks generated by these models, making it often difficult to label and hence interpret them. To overcome this interpretation problem many studies use structural VAR (SVAR) models, ${ }^{1}$ which allow shocks to be defined according to economic theory. This helps make SVAR model results potentially easier to interpret.

Although the SVAR model does solve the interpretation issue present in the reduced form model, it has a drawback in that it requires identification restrictions. This is due to the fact that only the reduced form parameters can be estimated consistently and efficiently. The structural parameters are estimated through their relation to the reduced form parameters. However, there are more structural parameters than reduced form parameters hence identification restrictions need to be imposed. ${ }^{2}$ A means of testing such restrictions is proposed by Lanne et al. (2010) and by Herwartz and Lütkepohl (2011). They modify the SVAR model to allow for a switching covariance matrix according to a Markov process. This makes it possible to test identifying restrictions, even when the model is just-identified in the traditional sense.

In this paper we re-examine the existence of stock price bubbles in the vein of Binswanger (2004) and Groenewold (2004). We begin by investigating whether the structural identification employed is appropriate by using the testing method proposed in Lanne et al. (2010) and Herwartz and Lütkepohl (2011). We then conduct empirical SVAR model simulations to further evaluate our findings and to determine whether there are any stock price bubbles present. In addition we extend the time series and number of countries analyzed compared with the earlier studies. We find that in the majority of cases the structural identification scheme is accepted. Empirical results show no signs in favor of any bubbles in stock prices.

This paper is structured as follows: section 2 gives a brief review of the relevant literature, section 3 introduces the SVAR and the MS-SVAR model. Section 4 discusses the MS-SVAR model findings and section 5 deals with the SVAR model empirical simulations. Finally, section 6 concludes.

\section{Literature Review}

This section briefly summarizes the main empirical contributions in the area of stock price bubbles and fundamentals relevant to our investigation. We discuss reduced form and structural models.

\footnotetext{
${ }^{1}$ Contributions in this area - investigating the relationship between share prices and other macro data by means of VAR or SVAR models - are mentioned in the following section concerning the relevant literature to our study.

${ }^{2}$ There need to be at least $K(K-1) / 2$ restrictions, where $K$ is the number of endogenous variables. For example, in a four-variable model there would need to be six restrictions in order to identify the structural shocks. Since these restrictions are just-identifying, and hence cannot be tested, they may not be innocuous.
} 
Studies applying VAR models to share prices and macroeconomic variables include some early work by Campbell and Shiller (1988), who try to forecast stock returns and find that using historical averages of real earnings is one of the most important predictors. Later applications include work by Gjerde and Saettem (1999) who analyze the relations among stock returns and many other macroeconomic variables for the Norwegian economy. They find that the real interest rate and real activity are important variables for explaining returns. Cheung and $\mathrm{Ng}$ (1998) conduct a similar analysis for several countries making use of a vector error correction (VEC) model to control for cointegrating relationships.

Structural models used in this area include work by Lee (1995), who finds that stock prices respond equally strong to both permanent and temporary shocks to dividends. In a follow up analysis, Lee (1998) introduces a non-fundamental component to the model and finds that stock prices tend to deviate from fundamentals only in the short-run and then gradually reach their price according to fundamentals. He therefore draws the conclusion that there are fads rather than bubbles present in stock prices. ${ }^{3}$ Lastrapes (1998) analyzes the effect of money supply shocks on stock and bond prices and concludes that there is a real liquidity effect for both. Rapach (2001) uses a four-variable SVAR model to characterize the effects of different macro shocks on stock prices. Relying on macro theory he imposes restrictions on the model to identify the structural shocks. He finds that the surge of share prices in the late 1990s was driven by the expansionary effects of these macro shocks and not due to any bubble components in stock prices. Slightly more recent publications are those by Binswanger (2004) and Groenewold (2004), both of whom use a bivariate SVAR model with industrial production and real share prices. They try to determine whether stocks are priced above their fundamentals, i.e. whether there are bubble components in stock prices. They conclude that from the mid 1990s this has indeed been the case.

\section{The Model}

In this paper we use a bivariate SVAR model as in Binswanger (2004) and Groenewold (2004). The two variables are the log of industrial production $\left(I P_{t}\right)$ and the log of real stock prices, $\left(s_{t}\right)$. Industrial production (as well as real GDP) is a commonly used proxy for real economic activity. Studies, such as James et al. (1985) and Chen et al. (1986), find that industrial production is a significant factor in explaining share prices. In this section we first describe the reduced form model, we then elaborate on the identification restriction and finish with a description of the MS-SVAR model and testing procedure.

The following $\operatorname{VAR}(p)$ model in first differences is considered:

$$
\Delta y_{t}=\nu+A_{1} \Delta y_{t-1}+A_{2} \Delta y_{t-2}+\cdots+A_{p} \Delta y_{t-p}+u_{t},
$$

where $\Delta y_{t}$ is a $(K \times 1)$ vector of the endogenous variables, in our case, $\Delta y_{t}=\left[\Delta I P_{t}, \Delta s_{t}\right]^{\prime}$, hence $K=2 . \Delta$ is the first difference operator (such that $\Delta y_{t}=y_{t}-y_{t-1}=(1-L) y_{t}$,

\footnotetext{
${ }^{3} \mathrm{~A}$ fad is defined as a gradual deviation of stock prices from their fundamentals rather than a sudden shift as in a bubble.
} 
where $L$ is the lag operator). $\nu$ is a $(2 \times 1)$ vector of constants, $A_{i}, i=1, \ldots, p$ are $(2 \times 2)$ parameter matrices and $u_{t}$ is a $(2 \times 1)$ vector of unobservable error terms with $E\left[u_{t}\right]=0$ and $E\left[u_{t} u_{t}^{\prime}\right]=\Sigma_{u}$, not necessarily diagonal (where $E$ denotes the expectation operator). The above equation can be rewritten as

$$
A(L) \Delta y_{t}=\nu+u_{t}
$$

where $A(L)=I_{K}-A_{1} L-A_{2} L^{2}-\cdots-A_{p} L^{p}$. Provided that $A(L)^{-1}$ exists, the Wold MA representation for the stationary $\Delta y_{t}$ process is

$$
\Delta y_{t}=\mu+\sum_{s=0}^{\infty} \Phi_{s} u_{t-s}=\mu+\Phi(L) u_{t}
$$

where $\mu=\left(I_{K}-A_{1}-A_{2}-\cdots-A_{p}\right)^{-1} \nu=A(1)^{-1} \nu, \Phi(L) \equiv A(L)^{-1}$ and $\Phi_{0}=$ $I_{K}$. Using the B-model, structural shocks are identified as $u_{t}=B \varepsilon_{t}$, where $B$ is the contemporaneous impact matrix. Further, it is usually assumed that $E\left[\varepsilon_{t} \varepsilon_{t}^{\prime}\right]=\Sigma_{\varepsilon}$ which is a diagonal covariance matrix (usually the identity matrix). Hence, the structural representation of the model is

$$
\Delta y_{t}=\mu+\sum_{s=0}^{\infty} \Psi_{s} \varepsilon_{t-s}=\mu+\Psi(L) \varepsilon_{t},
$$

where $\Psi_{i} \equiv \Phi_{i} B$, for $i=0,1,2, \ldots$. The accumulated long-run effects of the structural shocks over all periods are given by the long-run impact matrix, $\Psi \equiv \Phi B$, where $\Phi \equiv$ $\sum_{s=0}^{\infty} \Phi_{s}=A(1)^{-1}$.

\subsection{Identifying restrictions}

As already discussed, OLS estimation only yields consistent and efficient estimates of the reduced form parameters in (1). From the assumptions made above it follows that $\Sigma_{u}=B B^{\prime}$. Since the covariance matrix is symmetric, it has $K(K+1) / 2$ unique diagonal and off diagonal elements. The $B$ matrix on the other hand consists of $K^{2}$ elements. Hence, we need to impose $K^{2}-K(K+1) / 2=K(K-1) / 2$ restrictions to identify the structural parameters of the model. In our case this amounts to one restriction as $K=2$.

Restrictions can be imposed directly on the $B$ matrix or indirectly through the longrun impact matrix, $\Psi$ as is proposed by Blanchard and Quah (1989). We use the latter approach and restrict the upper right element, $\Psi_{1,2}$, of the long-run impact matrix to zero as done in Binswanger (2004) and Groenewold (2004). Hence,

$$
\Psi=\left[\begin{array}{cc}
\star & 0 \\
\star & \star
\end{array}\right]
$$

where $\star$ can take on any value. Consequently the structural shocks, $\varepsilon_{t}=\left[\varepsilon_{t}^{F}, \varepsilon_{t}^{N F}\right]^{\prime}$ can be interpreted as fundamental and non-fundamental shocks respectively. Hence, it is assumed that a fundamental shock can have a permanent effect on the economy and on 
the stock market, whilst a non-fundamental shock, although having a permanent effect on real stock prices, can only have a transitory effect on the economy.

This way the model is just-identified and hence the restriction in (5) cannot be tested conventionally. Therefore, we need to believe that we have captured the fundamental and non-fundamental components of stock prices. Indeed, it is for this reason that Groenewold (2004) prefers to label the shocks as macro and share market shocks instead of fundamental and non-fundamental shocks respectively. Clearly, it is beneficial to test this identification restriction so as to confirm the labeling of the shocks.

\subsection{The MS-SVAR model and testing procedure}

Testing structural identifying restrictions by means of a MS-SVAR in heteroskedasticity model is proposed by Lanne et al. (2010) and Herwartz and Lütkepohl (2011). This model is the same as the conventional VAR model given in (1) with the exception that the residuals are assumed to be normally and independently distributed conditional on being in a given state, $S_{t}$. In other words,

$$
u_{t} \mid S_{t} \sim \operatorname{NID}\left(0, \Sigma\left(S_{t}\right)\right)
$$

where $S_{t}$ follows a first order discrete valued Markov process. Normality is assumed so that it is possible to use maximum likelihood estimation to estimate the parameters. As is demonstrated in Lanne et al. (2010), this assumption is not restrictive and a wide class of unconditional distributions, other than just the normal, are captured. The discrete stochastic process $S_{t}$ is assumed to take on $M$ regimes with transition probabilities given by

$$
p_{i j}=P\left(S_{t}=j \mid S_{t-1}=i\right), \quad i, j=1, \ldots, M,
$$

which can be arranged in an $(M \times M)$ matrix of transition probabilities,

$$
P=\left[\begin{array}{cccc}
p_{11} & p_{12} & \cdots & p_{1 M} \\
p_{21} & p_{22} & \cdots & p_{2 M} \\
\vdots & \vdots & \ddots & \vdots \\
p_{M 1} & p_{M 2} & \cdots & p_{M M}
\end{array}\right]
$$

such that the probabilities add up to one row-wise. Hence, $p_{i M}=1-p_{i 1}-p_{i 2}-\cdots-$ $p_{i M-1}, i=1, \ldots, M$.

In order to test the identifying restriction it is necessary to decompose the covariance matrices in the following way:

$$
\Sigma(1)=B B^{\prime}, \quad \Sigma(2)=B \Lambda_{2} B^{\prime}, \quad \ldots \quad \Sigma(M)=B \Lambda_{M} B^{\prime},
$$

where $B$ is the contemporaneous impact matrix and $\Lambda_{i}=\operatorname{diag}\left(\lambda_{i 1}, \lambda_{i 2}\right), i=2, \ldots, M$ can be interpreted as relative variances. The underlying assumptions are that the contemporaneous effects matrix, $B$, stays constant across regimes and that shocks are orthogonal 
across regimes, i.e. $\Lambda_{i}, i=2, \ldots, M$ is diagonal. The assumption of a regime-invariant $B$ matrix means that impulse responses are constant throughout different time periods so that empirical simulation results are as in a common SVAR model. ${ }^{4}$ Orthogonality of the shocks implies that the covariance matrices are different across regimes, which is necessary to identify the $K^{2}$ parameters of the $B$ matrix and the $(M-1) K$ parameters of the diagonal $\Lambda_{i}, i=2, \ldots, M$ matrices. ${ }^{5}$ As shown in Proposition 1 of Lanne et al. (2010), provided that the pairwise diagonal elements of one of the $\Lambda_{i}, i=2, \ldots, M$ matrices are distinct, the $B$ matrix is identified up to sign changes and column ordering.

To estimate the model parameters we use the Expectation Maximization (EM) algorithm. This algorithm was initially popularized in Hamilton (1994) for the univariate case and was later extended to multivariate models in Krolzig (1997). The parameters of the decomposition in (8) are estimated using a non-linear optimization procedure in the Maximization step of the algorithm. Details of the estimation algorithm used in this paper are provided in the Appendix.

Once the EM algorithm has converged, standard errors of the point estimates of the unrestricted parameters are obtained through the inverse of the negative of the Hessian matrix evaluated at the optimum. The standard errors enable the use of Wald tests (and in addition we use LR tests) to determine whether the diagonal elements of at least one of the $\Lambda_{i}, i=2, \ldots, M$ matrices are distinct. The test distributions are asymptotically $\chi^{2}$, with their degrees of freedom depending on the number of joint hypotheses being tested.

Finally, provided that the $B$ matrix is identified up to changes in sign, any additional restrictions on it are over-identifying. Hence, the identifying restriction in (5) can be tested by means of a LR test. The test distribution is $\chi^{2}$ with a single degree of freedom since one restriction is being tested. The next section describes the data and the results of the MS-SVAR model.

\section{Testing the Restriction}

\subsection{The Data}

We obtain our data from the Federal Reserve Economic Data (FRED) database. The series consist of a seasonally adjusted industrial production index, a stock price index and a consumer price index (CPI), all of which are normalized to a base year of 2010 . The stock price series is converted to real terms by dividing by the percentage CPI, hence the CPI series is not used directly in the analysis. In addition, all series are in

\footnotetext{
${ }^{4} \mathrm{~A}$ state invariant $B$ matrix can be viewed as a restrictive assumption, though it can be tested provided that at least three Markov states are used. This is done by a standard likelihood ratio (LR) test, which has an asymptotically $\chi^{2}$ distributed test statistic with $(1 / 2) M K(K+1)-K^{2}-(M-1) K$ degrees of freedom.

${ }^{5}$ For example in case of two states we have two reduced form covariance matrices with $2 \times(K(K+$ $1) / 2)=K^{2}+K$ unique diagonal and off diagonal elements. This is enough to identify the $K^{2}$ elements of $B$ and the $K$ diagonal elements of $\Lambda_{2}$.
} 


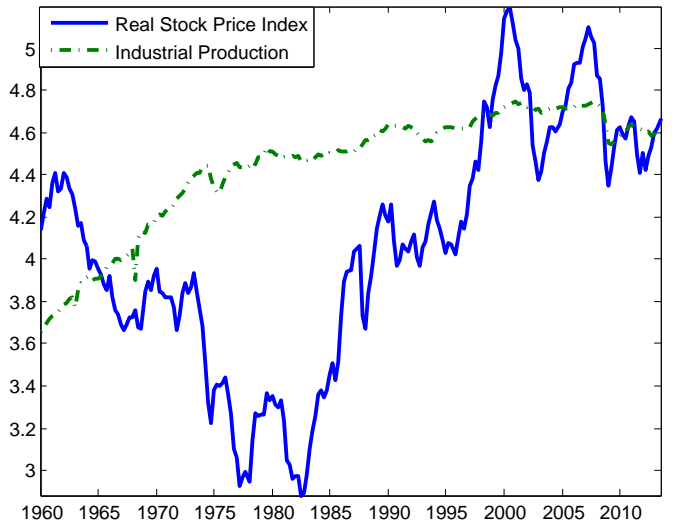

(a) France

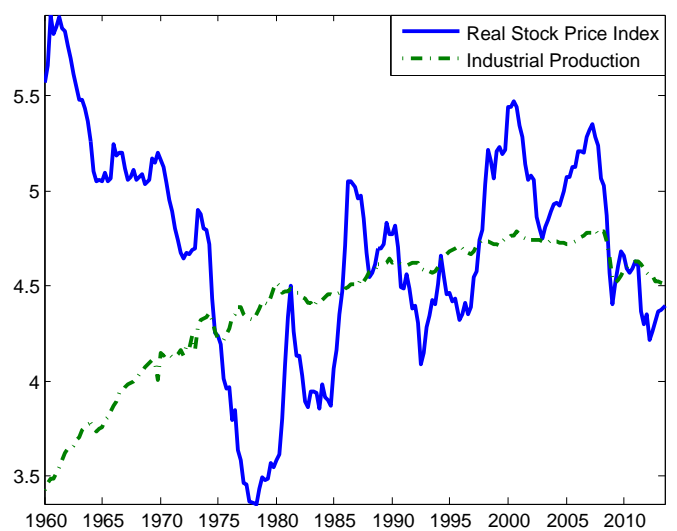

(c) Italy

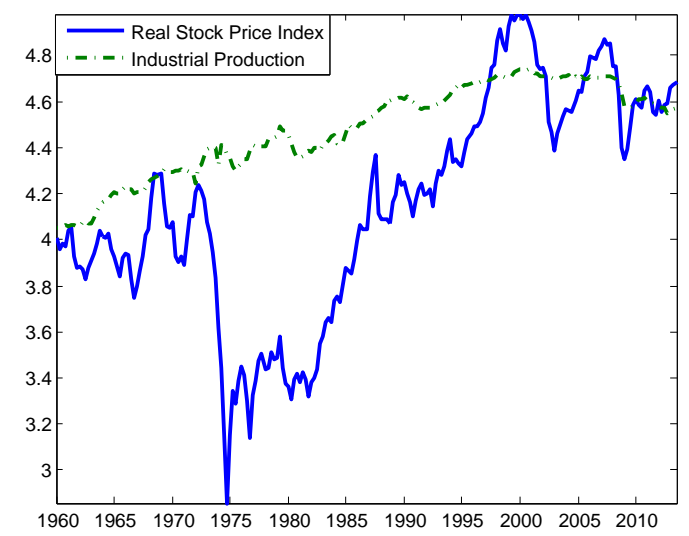

(e) UK

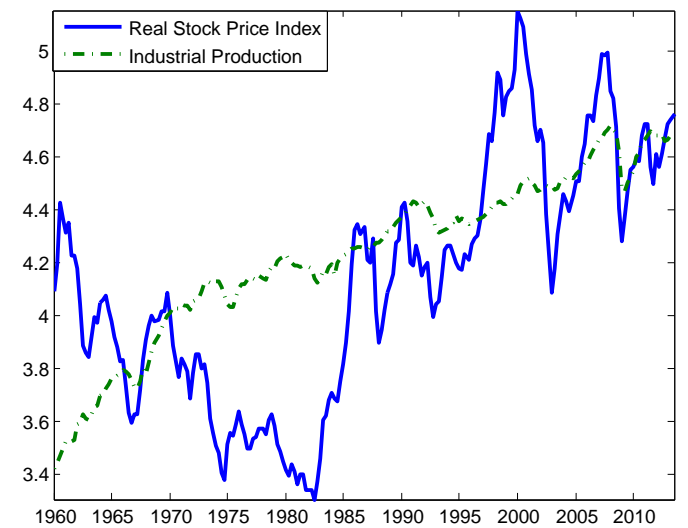

(b) Germany

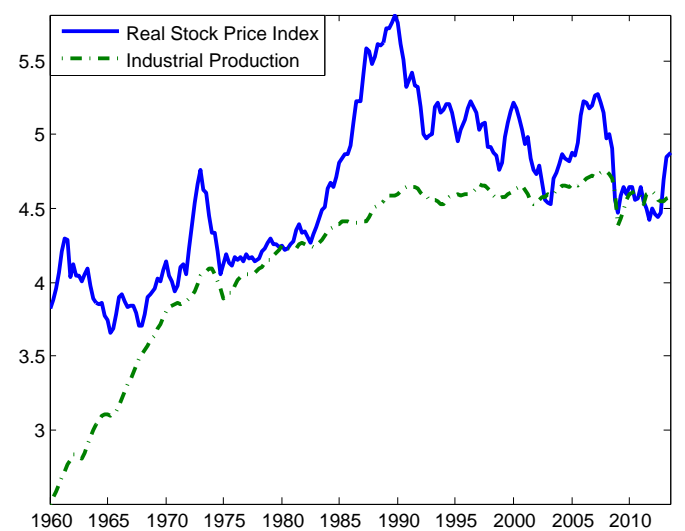

(d) Japan

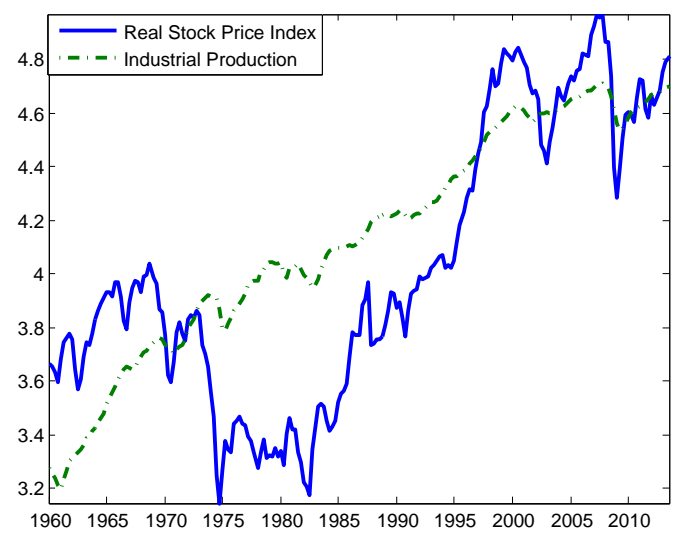

(f) US

Figure 2: Industrial production and real stock price series in log levels per country. 
logs. The data range is quarterly from 1960:I-2013:III; the frequency and the starting date being inline with Binswanger (2004) and Groenewold (2004).

We use data on France, Germany, Italy, Japan, the UK and the US. ${ }^{6}$ These are six of the G7 countries, they have high per capita GDP and their stock markets are well developed. Further, there are established authorities and agencies in these countries to ensure stock market transparency and limit insider trading. Hence, we consider them appropriate for our investigation. Figure 2 plots both variables for each country.

Augmented-Dickey-Fuller (ADF) unit root tests show that, for all countries, the levels series are integrated of order one, meaning that the first differences are stationary. When testing for cointegrating relationships on the levels VAR model using a constant and trend term, both the Saikkonen and Lütkepohl (2000) and the Johansen (1995) trace test usually cannot reject the null hypothesis of no cointegrating relations at the $1 \%$ level. At best there seems to be very weak evidence of cointegration among the variables. Hence, we use the standard VAR model in first differences, as given in equation (1). Multivariate conditional heteroskedasticity tests show that in most cases the null hypothesis of no heterskedasticity can be rejected at conventional significance levels. This lends support to using a model able to capture the heteroskedastic feature of the data, such as the MSVAR model already discussed. All of the above tests are carried out using the Lütkepohl and Krätzig (2004) JMulTi software.

\subsection{Model Specifications}

To select the appropriate number of states and lags for each data $\operatorname{model}^{7}$ we partially rely on the information criteria for Markov switching time series models developed by Psaradakis and Spagnolo (2006). These allow for joint determination of the state and lag orders and are also used by Herwartz and Lütkepohl (2011).We prefer however to select the number of lags based on residual Portmanteau tests. This usually gives a similar conclusion as we would obtain with the information criteria and we can further be confident that we have excluded residual autocorrelation.

For most data series the information criteria opt for two Markov states. We therefore, decide to use two-state models throughout the analysis. ${ }^{8}$ This allows for easier interpretation of the states and faster parameter estimation. Taking Portmanteau test results into account, we opt for a one-lag model for the data from France, Germany and Japan, a three-lag model for the data from Italy and the US and we model UK data with four lag orders.

\footnotetext{
${ }^{6}$ Japan and the US are also covered in Binswanger (2004).

${ }^{7}$ These are the MS-VAR models, or in other words the unrestricted models.

${ }^{8}$ In all cases the information criteria favor a MS model over a conventional VAR model, i.e. without any switching parameters. Further, log-likelihoods of switching models are considerably higher than those of non-switching models, which lends further support to using regime switching models.
} 


\subsection{MS-VAR Model Results}

The main parameters of interest for our analysis are the relative variance parameters, $\lambda_{i j}, i=2, \ldots, M, j=1,2$; which require to be distinct in at least one $\Lambda_{i}, i=2, \ldots, M$ matrix so that the contemporaneous impact matrix, $B$ in (8) is identified up to changes in sign. ${ }^{9}$ Other interesting parameters are the transition probabilities, $p_{i i}, i=1, \ldots, M$. These indicate how persistent a given state is and can be used to calculate the durations of states. These parameters and their standard errors along with the covariance matrices (scaled by $10^{-3}$ ) are presented in Table 1 .

The covariance matrices help classify the Markov states. Further information on the states is available from the smoothed probabilities. These display the probability of being in a given state at a particular time period. The ones for state one are shown in Figure 3. Naturally, the probability of being in state two is the mirror image of that of state one.

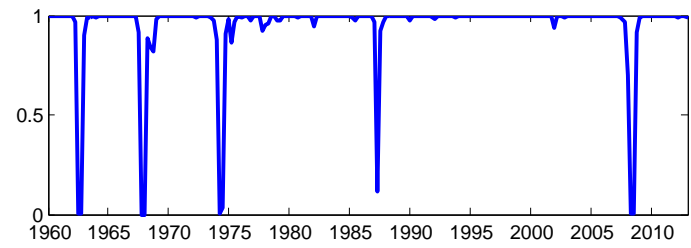

(a) France

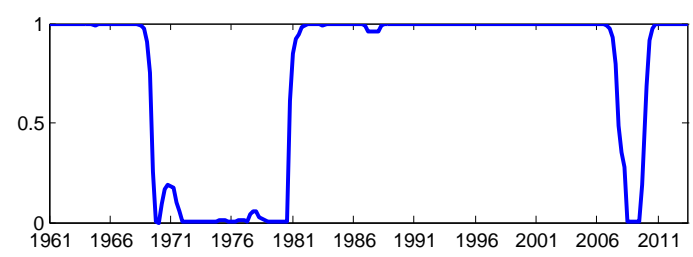

(c) Italy

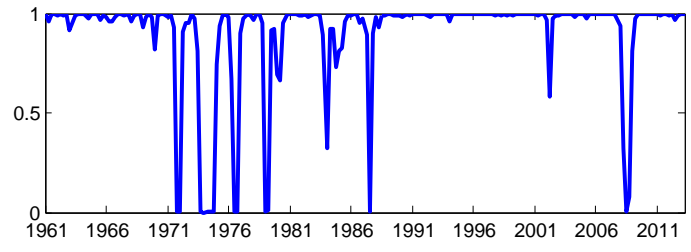

(e) UK

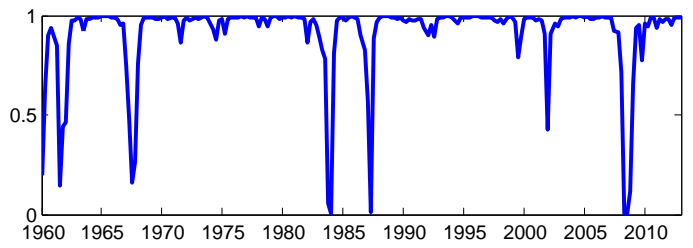

(b) Germany

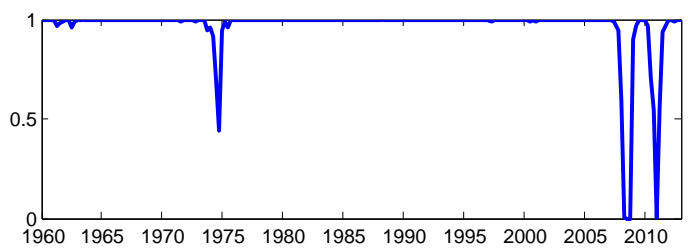

(d) Japan

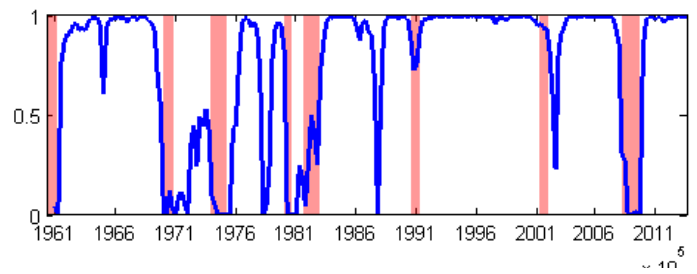

(f) US

Figure 3: Smoothed probabilities of state 1. US recession dates according to NBER dating given by the shaded bars.

\footnotetext{
${ }^{9}$ In our case it is required that $\lambda_{21} \neq \lambda_{22}$.
} 


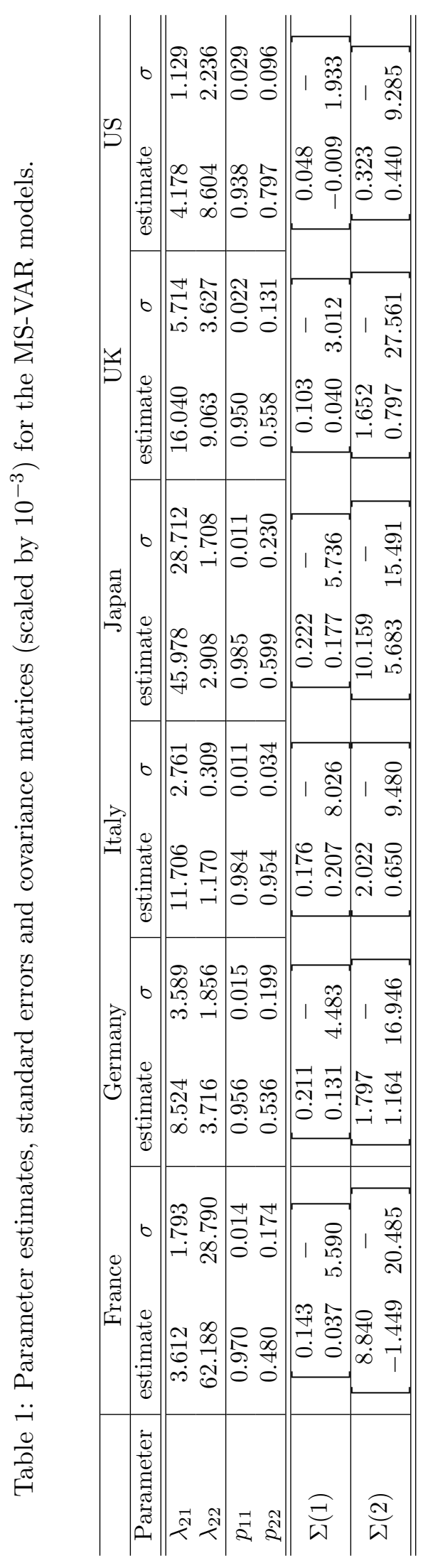


For all models ${ }^{10}$, the variances (the diagonal elements of the covariance matrices) increase with the state. In that sense state 2 can be thought of as depicting more volatile periods than state 1. This is also observed in the smoothed probabilities. Especially the ones for the US in Panel (f) of Figure 3, where US recession dates, according to NBER dating, are indicated by the shaded bars. Those smoothed probabilities show a clear tendency to switch to the more volatile state in times of recessions. This is in line with literature studying stock market volatility and stock returns. For instance, Schwert (1989) and Hamilton and Lin (1996) find that volatility increases are a result of economic downturns such as recessions or crises. Finally, since state 1 clearly has a much longer duration than the other state ${ }^{11}$, we can expect that it is more likely to be associated with non-recessionary periods as they tend to last longer. Hence, we interpret the first state as being the better or the non-recessionary state, while the second state is the more adverse or recessionary state.

We test, by means of a likelihood ratio (LR) and a Wald test, whether the pair of diagonal elements in $\Lambda_{2}$ are distinct. Recall that if they are we can be certain that the (state-invariant and unrestricted) $B$ matrix in (8) is identified up to changes in sign and column ordering. ${ }^{12}$ Results of these tests are shown in Table $2 .{ }^{13}$ Both tests yield the same conclusion at the $10 \%$ level. For most countries equality of the parameters can be rejected, except for Germany and the UK. For now however, let us assume that the contemporaneous matrix is identified in all cases and come back to this issue shortly.

Table 2: Null hypotheses and $p$-values for two and three state models.

\begin{tabular}{l|cc}
\hline$H_{0}: \lambda_{21}=\lambda_{22}$ & LR test & Wald test \\
\hline France & 0.000 & 0.042 \\
Germany & 0.213 & 0.200 \\
Italy & 0.000 & 0.000 \\
Japan & 0.032 & 0.130 \\
UK & 0.263 & 0.276 \\
US & 0.049 & 0.066 \\
\hline \hline
\end{tabular}

Assuming an identified $B$ matrix, the long-run restriction in (5) becomes overidentifying and can be tested. This is done by means of an LR test, $p$-values of which are summarized in Table $3 .{ }^{14}$ The identifying restriction is accepted at least at the $5 \%$

\footnotetext{
${ }^{10}$ Note that we refer to these models as MS-VAR models, although technically they have specific covariance matrices, as given in (8). This type of decomposition, where the $B$ matrix is held constant across states, is usually not very restrictive and can be tested when using more than two Markov states. In this analysis we only use two-state models, however, we test this decomposition using three-state models and find it to be supported in all cases.

${ }^{11}$ The exact formula for the duration of any of the $M$ states is $1 /\left(1-p_{i i}\right), i=1, \ldots, M$.

${ }^{12}$ This is referred to as being identified through heteroskedasticity in Lanne et al. (2010).

${ }^{13}$ The test distribution is asymptotically $\chi^{2}$ with one degree of freedom.

${ }^{14}$ Note that the null hypothesis is the long-run restriction given in (5), while the alternative is an
} 
level in most cases except for the data from Italy, and it is weakly accepted at the $1 \%$ level for Japan. Whether fundamental and non-fundamental shocks are indeed captured for Italy and Japan is further discussed in the next section on the SVAR model results.

Finally, recall that for Germany and the UK the contemporaneous matrix may not be identified through heteroskedasticity. As is argued in Lütkepohl and Velinov (2014), this would mean that the $p$-values for these countries in Table 3 are overstated. However, since these values are rather high (well above the $10 \%$ level) this may not be a serious problem. Of course, we cannot say how high they need to be in order to alleviate this issue completely. Given the current $p$-values we feel confident that the structural shocks in these countries can be labeled as fundamental and non-fundamental.

Table 3: $p$-values of LR tests of the long-run restriction in (5).

\begin{tabular}{cccccc}
\multicolumn{5}{c}{$H_{0}:(5) H_{1}:$ unrestricted state invariant $B$} \\
\hline France & Germany & Italy & Japan & UK & US \\
\hline 0.097 & 0.468 & 0.002 & 0.015 & 0.370 & 0.510 \\
\hline \hline
\end{tabular}

\subsection{A Robustness Check}

We conduct a robustness check to determine whether the above results are driven by the data period or the specific model used. Firstly, it can well be argued that the financial crisis marks a rather turbulent time. This event is captured by the smoothed probabilities of all country models making it a truly global occurrence. Such an event could influence our findings and hence, we exclude it when checking for robustness of the results. In other words we perform the above analysis using data until 2006:I. This way we are sure to avoid the financial crisis from being depicted in the smoothed probabilities.

Secondly, the specific type of MS model used could potentially influence the results. For instance, one may argue that, subject to the data used, other parameters aside from the covariance matrix could be regime switching. It is commonly observed that in times of high (low) volatility stock prices tend to go down (up). Hence, we can assume that the intercept vector could be state dependent. Further, the autoregressive parameters can also be subject to regime switches, however the case for them to switch is harder to justify and to interpret. In addition, switching autoregressive parameters may cause estimation problems since there are $M p K^{2}$ more parameters to be estimated. Hence, for our robustness check we use a model with a switching intercept term in addition to the switching covariance matrix. This model is as follows

$$
\Delta y_{t}=\nu\left(S_{t}\right)+A_{1} \Delta y_{t-1}+A_{2} \Delta y_{t-2}+\cdots+A_{p} \Delta y_{t-p}+u_{t},
$$

where $u_{t}$ has the same distributional assumption as in (6).

unrestricted (state invariant) $B$ matrix. 
The models used for the robustness analysis keep the same lag order as in the original specification. Portmanteau tests yield similar results as before, indicating that residual autocorrelation is avoided in most cases. The robustness analysis proceeds in the same manner as above. First, the parameters of the unrestricted MS models are estimated. Second, the distinction of the relative variance parameters is tested as in Table 2. Finally, the long-run restriction in (5) is tested. Results of this final step are presented in Table 4 along with the original $p$-values at the bottom for comparison.

Table 4: $p$-values of LR tests of the long-run restriction in (5) for different robustness specifications.

\begin{tabular}{|c|c|c|c|c|c|c|}
\hline & France & Germany & Italy & Japan & UK & US \\
\hline Short rage & 1.000 & 0.182 & $4.222 \times 10^{-5}$ & 0.015 & 0.382 & 0.194 \\
\hline Intercept & 0.058 & 0.381 & 0.040 & 0.010 & 0.315 & 0.998 \\
\hline Original & 0.097 & 0.468 & 0.002 & 0.015 & 0.370 & 0.510 \\
\hline
\end{tabular}

At a $5 \%$ critical level both robustness specifications reach the same conclusion as in the original specification. As before, the same identification issue is present concerning the contemporaneous matrix for Germany and the UK. However, their respective $p$-values seem sufficiently high so as not to warrant any concerns regarding the acceptance of the identification scheme. For Japan the identification scheme is again weakly accepted at the $1 \%$ level over all specifications. For Italy the identification restriction seems to be largely rejected over the different specifications and only weakly accepted when using a switching intercept. The potential consequences of rejecting the identification restriction are discussed in the next section. Finally, note that, when using the short range data for France, the restricted model gives a slightly higher log-likelihood than the unrestricted one. This is sometimes found in the MS literature, for instance in Droumaguet et al. (2014). It indicates strong evidence in favor of the identification scheme.

These results show that a globally significant event such as the financial crisis is not the driving force behind our original findings. Furthermore, the "simple" model we have used thus far delivers the same results as a more flexible model, allowing for the intercept term to switch. This justifies our initial use of a MS model in heteroskedasticity, which is the minimum requirement needed to test structural identification schemes.

\section{Empirical Results of the SVAR Model}

In this section we focus on the SVAR model, i.e. the conventional model without any regime switches. Our purpose is to firstly investigate further the structural shocks that we have studied with the MS-SVAR model above. We do this by means of impulse responses (IRs) and forecast error variance decompositions (FEVDs). Second, we seek to answer the question of whether there are bubbles in stock prices through historical 
decompositions (HDs). Since in most cases we find support for the long-run restriction in (5) in what follows we label the SVAR shocks as fundamental and non-fundamental. We keep the same lag orders as in the MS analysis above. This is again sufficient to remove any residual autocorrelation in the SVAR models.

\subsection{IRs and FEVDs}

In order to conduct a meaningful impulse response (IR) analysis we need to construct appropriate confidence intervals. In particular, since our data show signs of heteroskedasticity, which are evidenced in the above MS models, it is necessary to take this into account when computing the relevant confidence intervals. Accordingly, we use the fixed design wild bootstrap technique as in Goncalves and Kilian (2004) to construct the IR confidence intervals. The series are bootstrapped as

$$
\Delta y_{t}^{\star}=\widehat{\nu}+\widehat{A}_{1} \Delta y_{t-1}+\widehat{A}_{2} \Delta y_{t-2}+\cdots+\widehat{A}_{p} \Delta y_{t-p}+u_{t}^{\star},
$$

where $u_{t}^{\star}=\varphi_{t} \widehat{u}_{t}$ and where $\varphi_{t}$ is a random variable, independent of $y_{t}$ following a Rademacher distribution. In other words, $\varphi_{t}$ is either 1 or -1 with a $50 \%$ probability. The hat denotes estimated parameters. ${ }^{15}$

Since we are interested in identifying fundamental shocks to stock prices we focus on the accumulated response of real stock prices to a positive fundamental shock. This response is shown in Figure 4. Stock prices respond positively to such a shock as one would expect. This is also found by Binswanger (2004) and Groenewold (2004). Note that for Italy however, the long-run effect of the shock is insignificant at the $90 \%$ confidence level. Such a result is also found in Lütkepohl and Velinov (2014) when the identification scheme is rejected. This could mean that the identified shock is not necessarily a fundamental one to stock prices. However, for Japan, the shock does have a significant long-run impact, and hence, we can probably indeed classify it as being a fundamental shock. In addition, its response seems to be of a similar shape and magnitude as the other responses where the shocks are deemed to be of a fundamental nature. Indeed, the identification restriction for Japan is always waekly accepted at the $1 \%$ critical level.

We further investigate this issue by means of forecast error variance decompositions (FEVDs) of real stock prices. These tell us to what extent the structural shocks account for the forecast error of real stock prices. Results of these decompositions are given in Table $5^{16}$. The proportion of the forecast error variance of real stock prices accounted for by fundamental shocks is in most cases between $20 \%$ to $40 \%$. This number is lower for France ${ }^{17}$ and higher for the US, where fundamental shocks explain more than half of real stock price volatility. After about 10 quarters usually, the FEVDs stabilize meaning

\footnotetext{
${ }^{15}$ Note that, since this is a fixed design bootstrap method, in formulating the bootstrap series the lagged values are taken from the original data and not from the lagged bootstrap series.

${ }^{16}$ We are aware that our residuals exhibit signs of heteroskedasticity, however for the purpose of our analysis we conduct standard model simulations. Such simulations are also done in many of the papers discussed in section 2 where heteroskedasticity is not checked, even though stock price data is used.

${ }^{17}$ This could be due to its SVAR model having a single lag. For instance, when using three lags fundamental shocks account for more than $20 \%$ of real stock price volatility for all periods ahead.
} 


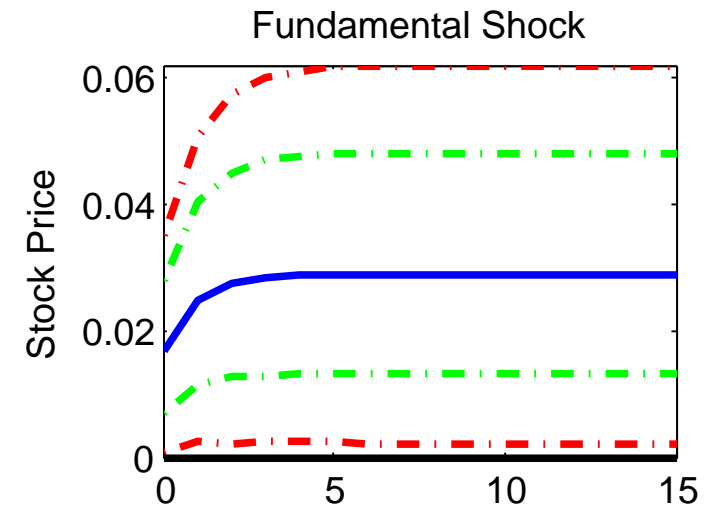

(a) France

Fundamental Shock

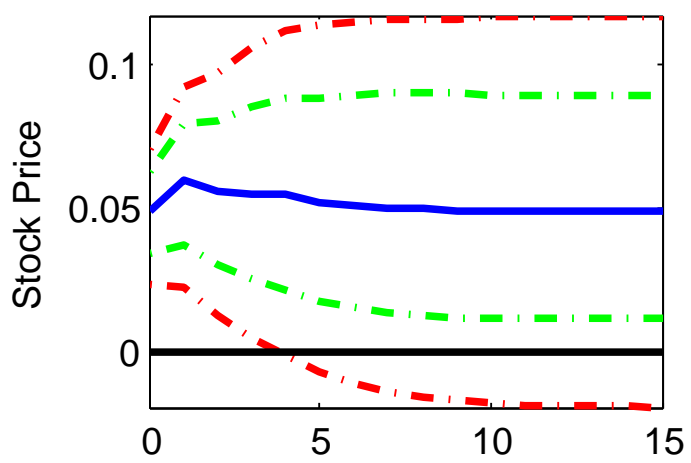

(c) Italy

Fundamental Shock

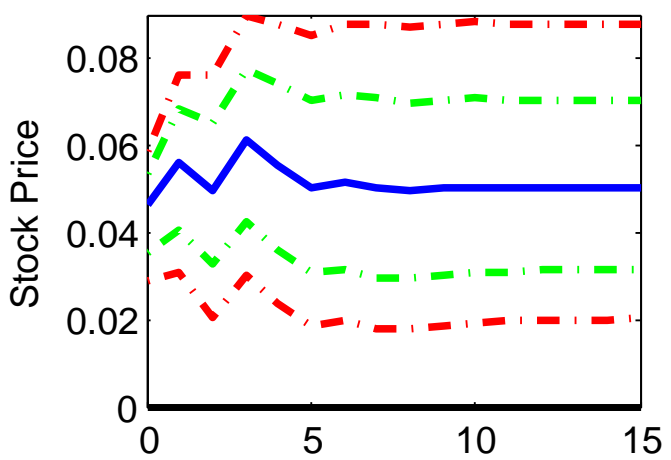

(e) UK

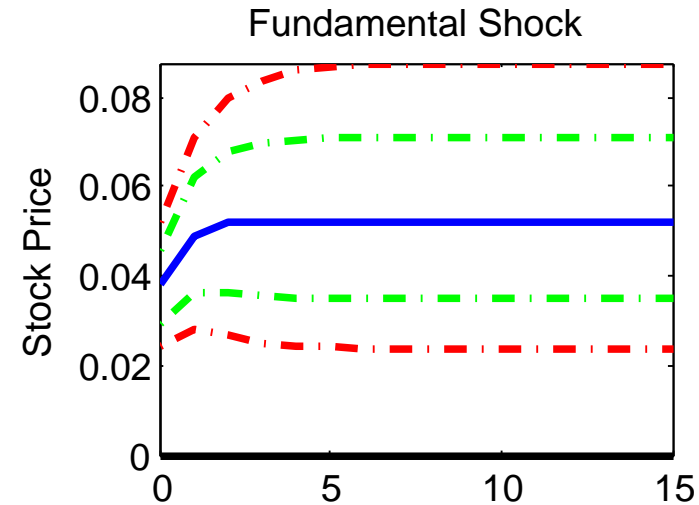

(b) Germany

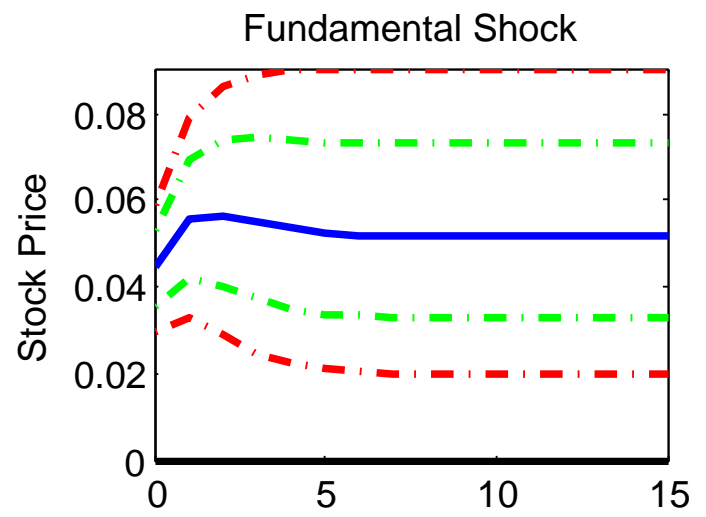

(d) Japan

Fundamental Shock

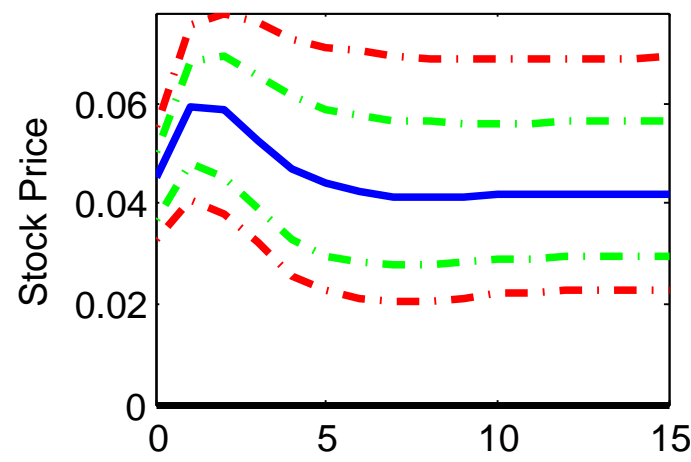

(f) US

Figure 4: Accumulated impulse responses of a fundamental shock to real stock prices. Broken lines indicate Efron confidence intervals according to the fixed design wild bootstrap technique at the 90 and 68 percentiles with 2000 replications. 
Table 5: FEVD's of the real stock price for all countries. Values are in percent

\begin{tabular}{|c|c|c|c|c|c|}
\hline \multirow[b]{2}{*}{ Quarters ahead } & \multicolumn{2}{|c|}{ Percentage of variance attributable to: } & \multirow[b]{2}{*}{ Quarters ahead } & \multicolumn{2}{|c|}{ Percentage of variance attributable to: } \\
\hline & $\begin{array}{l}\text { Fundamental } \\
\text { shock }\end{array}$ & $\begin{array}{l}\text { Non-fundamental } \\
\text { shock }\end{array}$ & & $\begin{array}{l}\text { Fundamental } \\
\text { shock }\end{array}$ & $\begin{array}{l}\text { Non-fundamental } \\
\text { shock }\end{array}$ \\
\hline France & & & Germany & & \\
\hline 1 & 4.31 & 95.69 & 1 & 26.18 & 73.82 \\
\hline 2 & 4.83 & 95.17 & 2 & 24.68 & 75.32 \\
\hline 3 & 4.87 & 95.13 & 3 & 24.39 & 75.61 \\
\hline 4 & 4.88 & 95.12 & 4 & 24.35 & 75.65 \\
\hline 5 & 4.88 & 95.12 & 5 & 24.34 & 75.66 \\
\hline 10 & 4.88 & 95.12 & 10 & 24.34 & 75.66 \\
\hline 15 & 4.88 & 95.12 & 15 & 24.34 & 75.66 \\
\hline 20 & 4.88 & 95.12 & 20 & 24.34 & 75.66 \\
\hline Italy & & & Japan & & \\
\hline 1 & 29.07 & 70.93 & 1 & 31.60 & 68.40 \\
\hline 2 & 26.58 & 73.42 & 2 & 29.71 & 70.29 \\
\hline 3 & 22.24 & 73.76 & 3 & 29.34 & 70.66 \\
\hline 4 & 25.51 & 74.49 & 4 & 29.34 & 70.66 \\
\hline 5 & 25.28 & 74.72 & 5 & 29.35 & 70.65 \\
\hline 10 & 25.25 & 74.75 & 10 & 29.36 & 70.64 \\
\hline 15 & 25.25 & 74.75 & 15 & 29.36 & 70.64 \\
\hline 20 & 25.25 & 74.75 & 20 & 29.36 & 70.64 \\
\hline UK & & & US & & \\
\hline 1 & 41.11 & 58.89 & 1 & 55.20 & 44.80 \\
\hline 2 & 37.82 & 62.18 & 2 & 53.50 & 46.50 \\
\hline 3 & 37.99 & 62.01 & 3 & 53.50 & 46.50 \\
\hline 4 & 39.24 & 60.76 & 4 & 53.86 & 46.14 \\
\hline 5 & 39.53 & 60.47 & 5 & 54.21 & 45.79 \\
\hline 10 & 39.69 & 60.31 & 10 & 54.30 & 45.70 \\
\hline 15 & 39.69 & 60.31 & 15 & 54.30 & 45.70 \\
\hline 20 & 39.69 & 60.31 & 20 & 54.30 & 45.70 \\
\hline
\end{tabular}

that the proportion of the forecast error variance explained by each shock stays at a constant level. Our findings for Japan and the US are similar to those in Binswanger (2004) when he uses his whole sample. ${ }^{18}$

Coming back to the issue regarding the identification of the structural shocks of Italy and Japan, it appears that their FEVDs are similar to those of the other countries. In particular, the second structural shock in Japan, according to IRs and FEVDs, behaves in the same way as shocks that are confirmed as being fundamental. Hence, we can confidently label it as such. The same shock for Italy on the other hand may not truly be a fundamental shock. However, for the purpose of investigating stock price bubbles we do label it as such when discussing the next simulation result.

\footnotetext{
${ }^{18}$ Since Binswanger (2004) argues for the existence of a structural break in the early 1980s he splits his sample in two and analyzes the FEVDs of both sub-samples as well as of the whole sample. In our analysis however, we choose to work with the whole sample since our purpose is to investigate the empirical results of the SVAR models having first tested whether the identifying restriction is supported by the data. Moreover, smoothed probabilities from our MS models show weak evidence at best of any globally significant event occurring around the early 1980s. If there is indeed such a phenomenon, it is less pronounced than the financial crisis.
} 


\subsection{HDs}

We perform historical decompositions (HDs) to answer our question of whether there are bubbles in stock prices. We do this by first obtaining the estimates of the structural shocks from $\widehat{\varepsilon}_{t}=\widehat{B}^{-1} \widehat{u}_{t}$. Then, after a specified starting period, we forecast the model through its moving average (MA) representation allowing only one shock at a time to be nonzero. In other words we obtain several forecast series, each of which is based on the influence of only a single specific shock. The exact formula for the decomposition is given as

$$
\Delta y_{T_{H}+j}-\hat{\mu}=\sum_{s=0}^{j-1} \hat{\Psi}_{s} \hat{\varepsilon}_{T_{H}+j-s}+\sum_{s=j}^{\infty} \hat{\Psi}_{s} \hat{\varepsilon}_{T_{H}+j-s},
$$

where the $\Psi$ s are the structural moving average coefficient matrices defined in (4) and $T_{H}$ is the starting period of the decomposition.

One of the first papers to use the HD technique is Burbidge and Harrison (1985) and it has arguably become an established methodology in empirical time series analysis. However, it suffers from a severe drawback in that the starting period of the decomposition, $T_{H}$ can be arbitrarily chosen and this influences the results to some extent. This problem is also noted by Binswanger (2004) and Groenewold (2004). In our analysis we let the decomposition start as soon as the MA forecast of the series closely matches the actual series ${ }^{19}$ which is in the latter part of the 1960s depending on different model lag orders. A close match between both series is necessary since, for better clarity, we integrate the historical series and the demeaned actual series forward so that both series are in levels, and any convergence problems would consequently be exacerbated due to this integration.

Results of the historical decompositions of real stock prices for all country models are displayed in Figure 5. The fundamental shock series, given by the thicker solid line, refers to the forecast series in which only fundamental shocks determine real stock prices, i.e. with non-fundamental shocks set to zero. The non-fundamental shock series is interpreted likewise. The demeaned actual real stock price series is given by the dashed line for comparison.

There are several apparent findings from these decompositions. First, real stock prices in all countries tend to be better explained by non-fundamental shocks. This result follows to some extent from the FEVDs above, where we find that in most cases non-fundamental shocks explain a larger percentage of the forecast volatility of real stock prices. Second, in all countries, except Japan, real stock prices have persistently been below their fundamentals throughout the 1970s until the mid 1980s or mid 1990s. This could be due to the oil crises occurring in the 1970s, which were globally significant events. Third, this undervaluation persists at most until the mid 1990s after which in all countries stock prices tend to be closer to their fundamental values.

Given these results we find no strong evidence of any bubbles in stock prices. Indeed, what we find can be characterized as a "negative" bubble, where stock prices tend

\footnotetext{
${ }^{19}$ The difference being less than $10^{-4}$.
} 


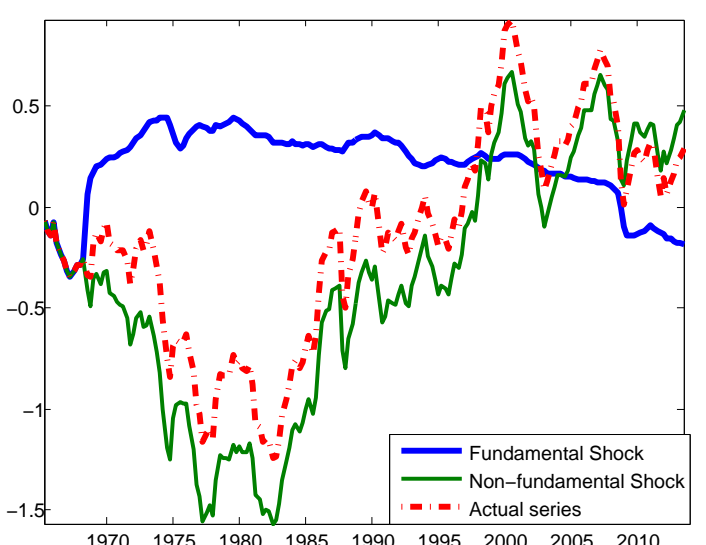

(a) France

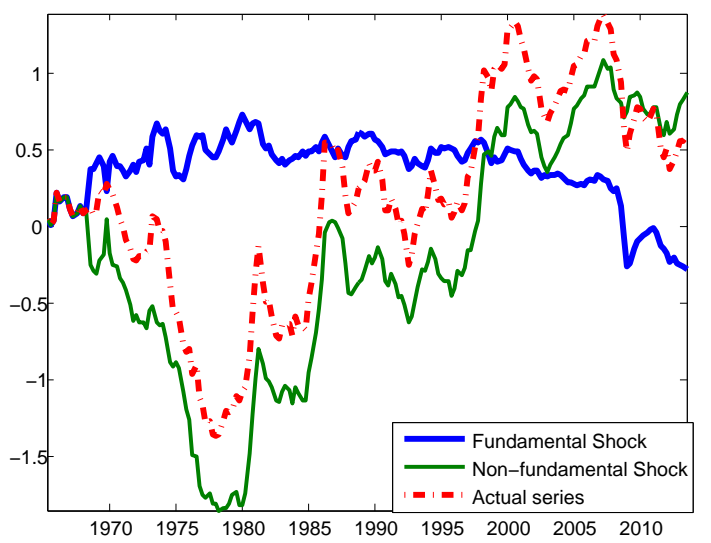

(c) Italy

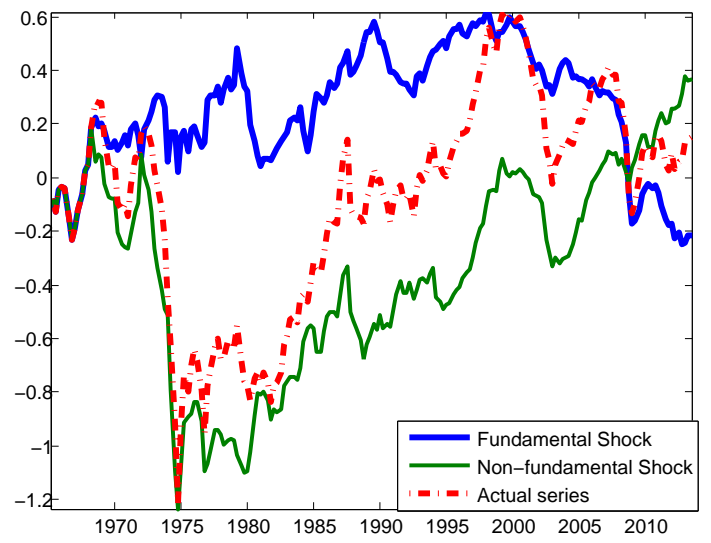

(e) UK

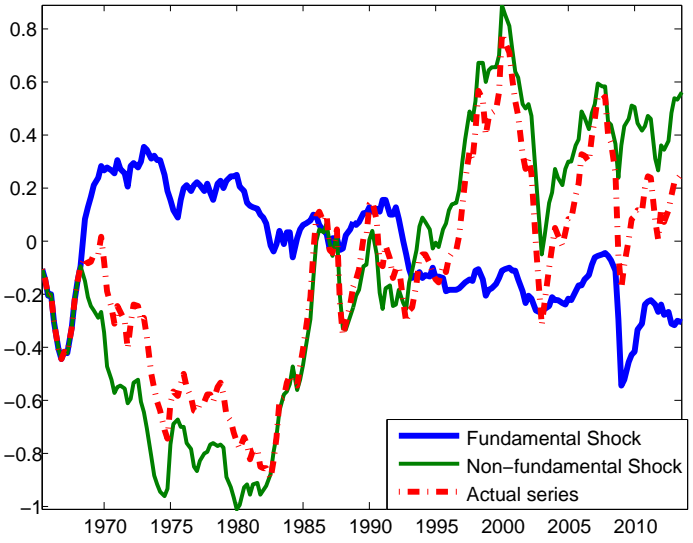

(b) Germany

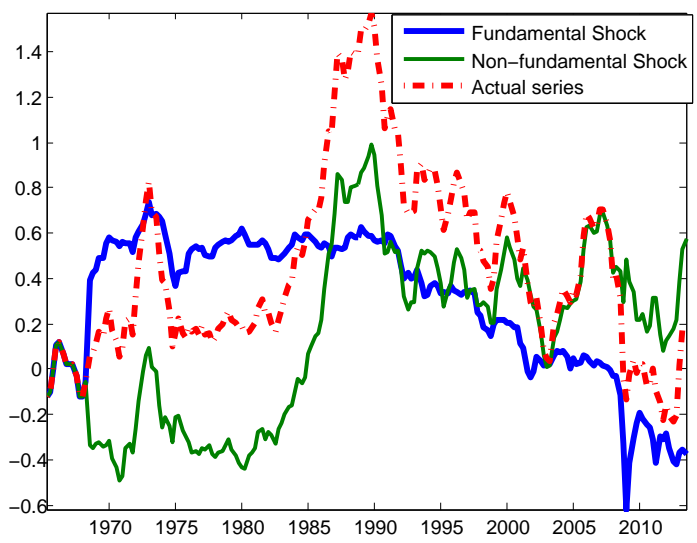

(d) Japan

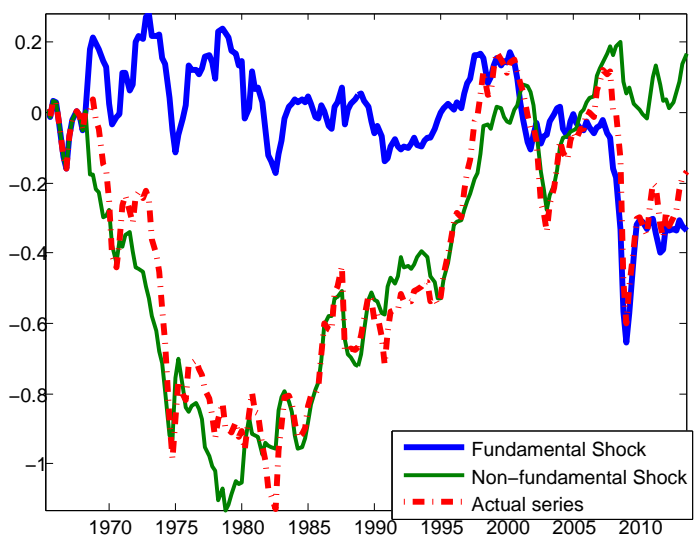

(f) US

Figure 5: Historical decompositions of the real stock price along with the actual demeaned stock price series. All series are in levels. 
to be undervalued for a while and then rapidly recover to be more in line with their fundamental values. This steep rise in stock prices, mainly throughout the mid 1990s, may have lead Binswanger (2004) and Groenewold (2004) to conclude the presence of a stock price bubble, which could be implied in Panel (a) of Figure 1. Our results are however more in agreement with those of Rapach (2001) and show this to be more likely a correction of a "negative" bubble. This finding is particularly strong for the US, where we can clearly see that after the financial crisis of the late 2000s real stock prices tend to move in tandem with their fundamentals. We therefore conclude that it is unlikely for bubbles to be present in stock prices.

\section{Conclusion}

In this paper we investigate whether there are any bubbles in stock prices as claimed in some previous studies. We do this by using a conventional bivariate SVAR model (considered by Binswanger (2004) and Groenewold (2004)) so that we can capture fundamental and non-fundamental shocks to stock prices. This model relies on an identification restriction so as to identify the shocks and to make it possible to estimate the structural parameters. Traditionally, this would make the model just-identified and hence the restriction would not be testable. We overcome this problem by using a Markov switching-SVAR (MS-SVAR) model in heteroskedasticity as in Lanne et al. (2010) and Herwartz and Lütkepohl (2011) to test the long-run restriction. This way we can be confident that our identification scheme is accepted by the data, and hence, our shock labeling is correct.

We use industrial production and real stock price data from France, Germany, Italy, Japan, the UK and the US. We model all regime switching models in two states and determine their lag orders so as to avoid residual autocorrelation. We find that the longrun restriction is supported at least at the $5 \%$ level for France, Germany, the UK and the US and is weakly supported at the $1 \%$ level for Japan. These results are consistent across several different specifications making our results robust. In addition, the identification restriction is supported for Italy when using a switching intercept term.

Having tested the identification restriction, we re-examine the structural shocks through traditional SVAR empirical simulations. In particular, we find as in Lütkepohl and Velinov (2014) that the long-run impulse response is less significant when the identification restriction is rejected. This is the case for Italy. Forecast error variance decompositions show that the shock deemed as fundamental behaves in a similar way across countries in explaining the forecast error variance of real stock prices. Hence, we feel confident in the labeling of the shocks for Japan, while there is no strong evidence in favor of the shock labeling for Italy. We do however presume to have identified the fundamental shocks for all countries so as to be able to investigate whether there are bubbles in stock prices.

As is common in this line of literature, we search for stock price bubbles by means of historical decompositions. Results of such decompositions show no evidence in favor of stock price bubbles in all countries under investigation. In fact, we tend to observe that 
stock prices are undervalued throughout the 1970s and 1980s leading us to conclude a possible "negative" bubble during that period. This event corrects itself in the mid 1990s, as is also implied by Rapach (2001), after which stock prices tend to move in tandem with their fundamentals. This correction could have been interpreted by Binswanger (2004) and Groenewold (2004) as a bubble in stock prices. For the US in particular we observe that after the financial crisis stock prices follow their fundamentals almost identically. We therefore find no evidence of any possible bubble components in stock prices.

\section{Appendix}

This is a technical appendix explaining the EM algorithm used in this paper in more detail. It is largely based on Krolzig (1997) and for more details the reader is referred to that work and to Chapter 22 of Hamilton (1994). Here $T$ denotes the sample size, $K$ the number of variables, $p$ the number of lags and $M$ the number of states.

\subsection{Definitions}

For the expectation step define

$$
\widehat{\xi}_{t \mid t}=\left[\begin{array}{c}
P\left(S_{t}=1 \mid \Delta Y_{t}\right) \\
P\left(S_{t}=2 \mid \Delta Y_{t}\right) \\
\vdots \\
P\left(S_{t}=M \mid \Delta Y_{t}\right)
\end{array}\right],
$$

an $(M \times 1)$ vector of conditional probabilities of being in a particular state at time period $t$ given all observations up to that time period, i.e. $\Delta Y_{t}=\left[\Delta y_{1}, \Delta y_{2}, \ldots, \Delta y_{t}\right]$. These are also referred to as the filtered probabilities of a MS model. Further, the conditional densities of an observation given a particular state, all past observations and all SVAR parameter estimates, $\theta$ are defined as

$$
\eta_{t}=\left[\begin{array}{c}
\left.P \Delta y_{t} \mid S_{t}=1, \Delta Y_{t-1}, \theta\right) \\
\left.P \Delta y_{t} \mid S_{t}=2, \Delta Y_{t-1}, \theta\right) \\
\vdots \\
P\left(\Delta y_{t} \mid S_{t}=M, \Delta Y_{t-1}, \theta\right)
\end{array}\right]=\left[\begin{array}{c}
\frac{1}{2 \pi|\Sigma(1)|^{1 / 2}} \exp \left\{-\frac{u_{t}^{\prime} \Sigma(1)^{-1} u_{t}}{2}\right\} \\
\frac{1}{2 \pi|\Sigma(2)|^{1 / 2}} \exp \left\{-\frac{u_{t}^{\prime} \Sigma(2)^{-1} u_{t}}{2}\right\} \\
\vdots \\
\frac{1}{2 \pi|\Sigma(M)|^{1 / 2}} \exp \left\{-\frac{u_{t}^{\prime} \Sigma(M)^{-1} u_{t}}{2}\right\}
\end{array}\right]
$$

Here $\theta$ consists of the vectorized SVAR parameters, i.e. $\nu, A_{i}, \Lambda_{j}, B, i=1, \ldots, p, j=$ $2, \ldots, M$.

For the maximization step define

- $\Delta y=\left[\Delta y_{1}^{\prime}, \ldots, \Delta y_{T}^{\prime}\right]^{\prime}$, a $(T K \times 1)$ vector of endogenous variables

- $\bar{Z}=\left[\mathbf{1}_{T}, \Delta Y_{-1}, \ldots, \Delta Y_{-p}\right]$, a $(T \times(1+K P))$ matrix 
- $\Delta Y_{-i}=\left[\Delta y_{1-i}, \ldots, \Delta y_{T-i}\right]^{\prime}, i=1, \ldots, p$, a $(T \times K)$ matrix of lagged regressors

- $\beta=\operatorname{vec}\left[\nu, A_{1}, \ldots, A_{p}\right]$, a $(K(K p+1) \times 1)$ vector of the parameters in (1)

- $u$, a $(T K \times 1)$ vector of residuals, $u_{i}, i=1, \ldots, T$ distributed according to (6).

Then (1) can be rewritten as

$\Delta y=\left(\bar{Z} \otimes I_{K}\right) \beta+u$,

where $\otimes$ denotes the Kronecker product.

\section{2 $\quad$ Starting Values}

- $\beta_{0}=\left[\bar{Z}^{\prime} \bar{Z} \otimes I_{K}\right]^{-1}\left(\bar{Z}^{\prime} \otimes I_{K}\right) \Delta y$

- $B_{0}=\left(U U^{\prime} / T\right)^{1 / 2}$, where $U$ is obtained from $u=\Delta y-\left(\bar{Z} \otimes I_{K}\right) \beta_{0}$, where $u=$ $\operatorname{vec}(U)$

- $P_{0}=\mathbf{1}_{M} \mathbf{1}_{M}^{\prime} / M, \mathbf{1}_{M}$ is an $(M \times 1)$ vector of ones

- $\xi_{0 \mid 0}=\mathbf{1}_{M} / M$

- $\Lambda_{2}=i \times I_{K}, \Lambda_{3}=i^{2} \times I_{K}, \ldots, \Lambda_{M}=i^{M} \times I_{K}, i=2, \ldots$ Different values of $i$ are used to determine which gives the highest sensible log-likelihood.

\subsection{Expectation Step}

Calculate the filtered probabilities, (11) from (12) as

$$
\widehat{\xi}_{t \mid t}=\frac{\widehat{\eta}_{t} \odot \widehat{\xi}_{t \mid t-1}}{\mathbf{1}_{M}^{\prime}\left(\widehat{\eta}_{t} \odot \widehat{\xi}_{t \mid t-1}\right)},
$$

where

$$
\widehat{\xi}_{t \mid t-1}=\widehat{P}^{\prime} \widehat{\xi}_{t-1 \mid t-1},
$$

for $t=1, \ldots, T$. This generates an $(M \times 1)$ vector of conditional probabilities for each time period. Here $\odot$ denotes element-by-element multiplication and $\widehat{P}$ is defined as in (7). The sum of the denominators for $t=1, \ldots, T$ in (13) is the likelihood of the MSSVAR model as noted in Hamilton (1994). Using the estimated filtered probabilities, the smoothed probabilities, conditional on all observations up to time $T, P\left(S_{t}=i \mid \Delta Y_{T}\right), i=$ $1, \ldots, M$ are estimated as

$$
\widehat{\xi}_{t \mid T}=\left[\widehat{P}\left(\widehat{\xi}_{t+1 \mid T} \oslash \widehat{\xi}_{t+1 \mid t}\right)\right] \odot \widehat{\xi}_{t \mid t}
$$

for $t=T-1, \ldots, 0$ where $\widehat{\xi}_{T \mid T}$ is taken from the last iteration in (13). The symbol $\oslash$ denotes element-by-element division. 


\subsection{Maximization Step}

Calculate $\rho=\operatorname{vec}(P)$ using (13), (14) and (15) as

$$
\widehat{\rho}=\widehat{\xi}^{(2)} \oslash\left(\mathbf{1}_{M} \otimes \widehat{\xi}^{(1)}\right),
$$

where $\widehat{\xi}^{(1)}=\left(\mathbf{1}_{M}^{\prime} \otimes I_{M}\right) \widehat{\xi}^{(2)}, \widehat{\xi}^{(2)}=\sum_{t=0}^{T-1} \widehat{\xi}_{t \mid T}^{(2)}$ and

$$
\widehat{\xi}_{t \mid T}^{(2)}=\operatorname{vec}(\widehat{P}) \odot\left[\left(\widehat{\xi}_{t+1 \mid T} \oslash \widehat{\xi}_{t+1 \mid t}\right) \otimes \widehat{\xi}_{t \mid t}\right] .
$$

Using (15), estimate $B$ and $\Lambda_{i}, i=2, \ldots, M$ by optimizing

$$
\begin{aligned}
l\left(B, \Lambda_{2}, \ldots, \Lambda_{M}\right) & =T \log |\operatorname{det}(B)|+\frac{1}{2} \operatorname{tr}\left(\left(B B^{\prime}\right)^{-1} \widehat{U} \widehat{\Xi}_{1} \widehat{U}^{\prime}\right) \\
& +\sum_{m=2}^{M}\left[\frac{\widehat{T}_{m}}{2} \log \left(\operatorname{det}\left(\Lambda_{m}\right)\right)+\frac{1}{2} \operatorname{tr}\left(\left(B \Lambda_{m} B^{\prime}\right)^{-1} \widehat{U} \widehat{\Xi}_{m} \widehat{U}^{\prime}\right)\right]
\end{aligned}
$$

where $\widehat{U}$ is obtained from $\widehat{u}=\Delta y-\left(\bar{Z} \otimes I_{K}\right) \widehat{\beta}$ where $\widehat{u}=\operatorname{vec} \widehat{U}, \widehat{\Xi}_{m}=\operatorname{diag}\left(\widehat{\xi}_{m 1 \mid T}, \ldots, \widehat{\xi}_{m T \mid T}\right)$, the smoothed probabilities of regime $m$ and $\widehat{T}_{m}=\sum_{t=1}^{T} \widehat{\xi}_{m t \mid T}$. To avoid singularity a lower bound of 0.01 is imposed on the diagonal elements of the $\Lambda_{m}, m=2, \ldots, M$ matrices. Using the estimates from (17), the updated covariance matrices are then derived as in (8) as

$$
\widehat{\Sigma}(1)=\widehat{B} \widehat{B}^{\prime}, \quad \widehat{\Sigma}(2)=\widehat{B} \widehat{\Lambda}_{2} \widehat{B}^{\prime}, \quad \ldots \quad \widehat{\Sigma}(M)=\widehat{B} \widehat{\Lambda}_{M} \widehat{B}^{\prime} .
$$

Using (15) and (18) calculate the remaining SVAR parameters as

$$
\widehat{\beta}=\left[\sum_{m=1}^{M}\left(\bar{Z}^{\prime} \widehat{\Xi}_{m} \bar{Z}\right) \otimes \widehat{\Sigma}^{-1}(m)\right]^{-1}\left[\sum_{m=1}^{M}\left(\bar{Z}^{\prime} \widehat{\Xi}_{m}\right) \otimes \widehat{\Sigma}^{-1}(m)\right] \Delta y .
$$

Obtain a new $\widehat{U}$ using $\widehat{\beta}$ from (19) and keep on re-estimating (17), (18) and (19) until a convergence criterion is met.

Finally, using (15),

$$
\widehat{\xi}_{0 \mid 0}=\widehat{\xi}_{0 \mid T} .
$$

\subsection{Convergence}

The expectation and maximization steps are iterated until convergence. Recall, the log-likelihood of the MS-SVAR model is given by

$$
l\left(\theta \mid \Delta Y_{T}\right)=\sum_{t=1}^{T} \ln \left(\mathbf{1}_{M}^{\prime}\left(\widehat{\eta}_{t} \odot \widehat{\xi}_{t \mid t-1}\right)\right) .
$$


We use the absolute change in the log-likelihood as a convergence criterion in the maximization step and for the EM algorithm as a whole, i.e.

$$
\Delta=\left|l(\bullet)^{j+1}-l(\bullet)^{j}\right|
$$

where $l(\bullet)^{j}$ is the log-likelihood given by (17) or (21) for the $j$-th iteration. Convergence is satisfied when $\Delta \leq 10^{-6}$ or after a specified maximum number of iterations.

\subsection{Switching Intercept}

The model with a switching intercept term as well as a switching covariance matrix, given in (9) is easily calculated with a small modification to the $\bar{Z}$ matrix. Namely,

$$
\bar{Z}_{m}=\left[\mathbf{1}_{T} \otimes \iota_{m}^{\prime}, \Delta Y_{-1}, \ldots, \Delta Y_{-p}\right], m=1, \ldots, M,
$$

where $\iota_{m}$ is the $m$-th column of the $M$-dimensional identity matrix. The above matrix is of a $(T \times(M+K P))$ dimension. Hence, $\widehat{\beta}=\operatorname{vec}\left[\widehat{\nu}(1), \ldots, \widehat{\nu}(M), \widehat{A}_{1}, \ldots, \widehat{A}_{p}\right]$ in $(19)$.

\section{7 $\quad$ Standard Errors}

Upon convergence of the EM algorithm, the optimal values of $\beta, B, \Lambda_{m}, m=2, \ldots, M, \xi_{0 \mid 0}$ and the $M(M-1)$ unrestricted parameters in $P$ are used in a function to calculate the log-likelihood in (21) from (12), (13) and (14). Using this function, standard errors of all unrestricted parameters are obtained by the inverse of the negative of the Hessian matrix.

\section{References}

Binswanger, M. (2004). How important are fundamentals? Evidence from a structural VAR model for the stock markets in the US, Japan and Europe. Journal of International Financial Markets, Institutions \&3 Money 14(2), 185-201.

Blanchard, O. and D. Quah (1989). The dynamic effects of aggregate demand and supply disturbances. The American Economic Review 79(4), 655-673.

Burbidge, J. and A. Harrison (1985). An historical decomposition of the great depression to determine the role of money. Journal of Monetary Economics 16(1), 45-54.

Campbell, J. and R. Shiller (1988). Stock prices, earnings, and expected dividends. Journal of Finance 43(3), 661-676.

Chen, N., R. Roll, and S. Ross (1986). Economic forces and the stock market. Journal of Business 59(3), 383-403.

Cheung, Y. and L. Ng (1998). International evidence on the stock market and aggregate economic activity. Journal of Empirical Finance 5(3), 281-296. 
Droumaguet, M., A. Warne, and T. Woźniak (2014). Granger causality and regime inference in bayesian markov-switching vars. Technical report, Discussion Paper, EUI Florence.

Gjerde, Ø. and F. Saettem (1999). Causal relations among stock returns and macroeconomic variables in a small, open economy. Journal of International Financial Markets, Institutions and Money 9(1), 61-74.

Goncalves, S. and L. Kilian (2004). Bootstrapping autoregressions with conditional heteroskedasticity of unknown form. Journal of Econometrics 123(1), 89-120.

Groenewold, N. (2004). Fundamental share prices and aggregate real output. Applied Financial Economics 14(9), 651-661.

Hamilton, J. and G. Lin (1996). Stock market volatility and the business cycle. Journal of Applied Econometrics 11(5), 573-593.

Hamilton, J. D. (1994). Time Series Analysis, Volume 2. Princeton university press.

Herwartz, H. and H. Lütkepohl (2011). Structural vector autoregressions with markov switching: Combining conventional with statistical identification of shocks. Technical report, Discussion Paper, EUI Florence.

James, C., S. Koreisha, and M. Partch (1985). A VARMA analysis of the causal relations among stock returns, real output, and nominal interest rates. Journal of Finance 40(5), 1375-1384.

Johansen, S. (1995). Likelihood-based inference in cointegrated vector autoregressive models. Cambridge Univ Press.

Krolzig, H. (1997). Markov-Switching Vector Autoregressions-Modelling.

Lanne, M., H. Lütkepohl, and K. Maciejowska (2010). Structural vector autoregressions with markov switching. Journal of Economic Dynamics and Control 34(2), 121-131.

Lastrapes, W. (1998). International evidence on equity prices, interest rates and money. Journal of International Money and Finance 17(3), 377-406.

Lee, B. (1995). The response of stock prices to permanent and temporary shocks to dividends. Journal of Financial and Quantitative Analysis, 1-22.

Lee, B. (1998). Permanent, temporary, and non-fundamental components of stock prices. Journal of Financial and Quantitative Analysis 33(1), 1-32.

Lütkepohl, H. and M. Krätzig (Eds.) (2004). Applied Time Series Econometrics. Cambridge: Cambridge University Press.

Lütkepohl, H. and A. Velinov (2014). Structural vector autoregressions: Checking identifying long-run restrictions via heteroskedasticity. Technical report, Discussion Papers, DIW Berlin. 
Psaradakis, Z. and N. Spagnolo (2006). Joint determination of the state dimension and autoregressive order for models with Markov regime switching. Journal of Time Series Analysis 27(5), 753-766.

Rapach, D. (2001). Macro shocks and real stock prices. Journal of Economics and Business 53(1), 5-26.

Saikkonen, P. and H. Lütkepohl (2000). Testing for the cointegrating rank of a var process with structural shifts. Journal of Business 83 Economic Statistics 18(4), 451-464.

Schwert, G. (1989). Why does stock market volatility change over time? Journal of Finance 44(5), 1115-1153. 